\title{
MPZL1 promotes tumor cell proliferation and migration via activation of Src kinase in ovarian cancer
}

\author{
DANNI CHEN, LEI CAO and XIAOJIE WANG \\ Department of Obstetrics and Gynecology, Tongren Hospital, \\ Shanghai Jiao Tong University School of Medicine, Shanghai 200336, P.R. China
}

Received March 10, 2018; Accepted May 28, 2019

DOI: $10.3892 /$ or.2019.7199

\begin{abstract}
Tumor metastasis is the leading cause of mortality in patients with advanced ovarian cancer. Myelin protein zero like 1 (MPZL1) is a transmembrane glycoprotein that promotes migration of hepatocellular carcinoma cells and is involved in extracellular matrix-induced signal transduction. However, the functional role of MPZL1 in ovarian cancer has not been well elucidated. The present study conducted western blotting, phase-contrast imaging and immunohistochemistry to reveal the functions of MPZL1 in ovarian cancer. The present study demonstrated that the expression levels of MPZL1 were associated with malignant features of ovarian cancer. Furthermore, overexpression of MPZL1 significantly promoted cell proliferation, migration and invasion of ovarian cancer cells. Conversely, MPZL1 depletion by short hairpin RNA inhibited migration and invasion of ovarian cancer cells. In addition, this study demonstrated that phosphorylation of Src kinase was increased upon MPZLI overexpression. Additionally, phosphorylation and activation of pro-metastatic proteins p130 and cortactin were induced by phosphorylated Src kinase. Collectively, these findings indicated that MPZL1 may be a novel pro-metastatic gene, which promotes tumor cell proliferation and migration through Src-mediated phosphorylation of p130 and cortactin in ovarian cancer.
\end{abstract}

\section{Introduction}

Ovarian cancer has the highest mortality rate among gynecological malignancies worldwide $(1,2)$. Tumor metastasis is a complex biological process involving cell signaling, regulation of cell proliferation, motility and invasion; in

Correspondence to: Dr Xiaojie Wang, Department of Obstetrics and Gynecology, Tongren Hospital, Shanghai Jiao Tong University School of Medicine, 1111 Xianxia Road, Shanghai 200336, P.R. China

E-mail: wangxiaojie2008@hotmail.com

Key words: MPZL1, proliferation, migration, Src kinase, ovarian cancer addition, metastasis is the primary cause of death in patients with advanced ovarian cancer and is closely associated with unfavorable outcomes and poor prognosis (3-7). Recently, the rat sarcoma-mitogen-activated protein kinase, phosphoinositide 3-kinase-protein kinase $\mathrm{B}$ and janus kinase-signal transducer and activator of transcription signaling pathways have been reported to be associated with tumor metastasis and invasion in ovarian cancer (8). Furthermore, the tumor microenvironment, which includes stromal cells, extracellular matrix components and exosomes, can establish a communication circuit that enhances cancer cell invasion and metastasis via reciprocal signaling (9). However, the molecular mechanisms underlying ovarian cancer metastasis are currently not well elucidated (10).

Myelin protein zero like 1 (MPZL1), also known as protein zero-related, is a hyperphosphorylated transmembrane glycoprotein involved in extracellular matrix-induced signal transduction (11-15). Previous studies have demonstrated that MPZL1 promotes hepatocellular carcinoma cell migration through the Src tyrosine kinase, and may be involved in adhesion-dependent signaling $(11,14,16)$. Furthermore, MPZL1 forms a complex with the growth factor receptor-bound protein 2 adaptor and tyrosine-protein phosphatase non-receptor type 11 phosphatase, and is involved in cell adhesion in human epidermal growth factor receptor 2-positive breast cancer cells (12). Additionally, as a major receptor of concanavalin A, MPZL1 has an important role in cell signaling via c-Src (13). However, the functional role and clinical implications of MPZL1 in ovarian cancer are largely unknown.

The present study demonstrated that amplification of MPZL1 was associated with malignant features of ovarian cancer, and promoted tumor cell proliferation, migration and invasion. Furthermore, overexpression of MPZL1 significantly promoted cell growth and metastasis of ovarian cancer. Conversely, knockdown of MPZL1 via short hairpin RNA (shRNA) attenuated proliferation and migration of ovarian cancer cells. This study also demonstrated that MPZL1 overexpression-induced activation of Src kinase mediated the phosphorylation and activation of cortactin and p130 in ovarian cancer. Taken together, these findings suggested that MPZL1 may be considered a novel pro-metastatic gene that promotes tumor cell proliferation and migration via activation of Src kinase in ovarian cancer. 


\section{Materials and methods}

Collection of ovarian cancer specimens. The present study was approved in January 2017 by the Ethics Committee of the Tongren Hospital, Shanghai Jiao Tong University School of Medicine. The collection of ovarian cancer specimens was performed in conformity to ethical standards. All participants provided written informed consent. The tissues were obtained during surgery and were fixed with $4 \%$ paraformaldehyde (Beijing Solarbio Science \& Technology Co., Ltd.) for $24 \mathrm{~h}$ at room temperature and embedded in paraffin. Specimens were obtained from 78 patients (age, 29-77 years, including 16 benign, 16 borderline and 46 malignant patients) with epithelial ovarian cancer who had not previously undergone treatment; the benign group consisted of patients with a benign ovarian tumor. No distant metastasis was detected in the selected patients prior to surgery. Detailed pathological data, including histological type, tumor size, tumor stage and lymph node metastasis, were obtained and summarized. The tumor stage was defined using the 8th edition of the Union for International Cancer Control-tumor node metastasis classification system (17).

Reagents and cell lines. The human ovarian cancer cell lines 293T, HO8910, SKOV3, HEY and TOV-21G were purchased from American Type Culture Collection and were cultured in RPMI-1640 medium (Gibco; Thermo Fisher Scientific, Inc.) supplemented with $10 \%$ fetal bovine serum (FBS; Gibco; Thermo Fisher Scientific, Inc.) and 1\% penicillin/streptomycin (Gibco; Thermo Fisher Scientific, Inc.).

Virus production and infection. 293T cells (60\% confluent) in a $10 \mathrm{~cm}$ dish were co-transfected with $5 \mu \mathrm{g}$ lentiviral constructs (pLenti 7.3; Invitrogen; Thermo Fisher Scientific, Inc.), $5 \mu \mathrm{g}$ plasmid $\Delta 8.9$ (Invitrogen; Thermo Fisher Scientific, Inc.) and $3 \mu \mathrm{g}$ plasmid vesicular stomatitis virus $\mathrm{G}$ (Invitrogen; Thermo Fisher Scientific, Inc.) using Lipofectamine ${ }^{\circledR} 2000$ (Invitrogen; Thermo Fisher Scientific, Inc.). Cells were incubated at $37^{\circ} \mathrm{C}$ and the medium was replaced after $12 \mathrm{~h}$. Virus-containing medium was collected $48 \mathrm{~h}$ post-transfection and supplemented with $8 \mu \mathrm{g} / \mathrm{ml}$ polybrene to infect target cells in 6-well dishes $\left(60 \%\right.$ confluent) at $37^{\circ} \mathrm{C}$. Infected cells were selected with $3 \mu \mathrm{g} / \mathrm{ml}$ puromycin at $37^{\circ} \mathrm{C}$ for $\geq 1$ week post-infection. The lentiviral shRNA vectors targeting $M P Z L 1$ and scrambled control shRNA were purchased from Open Biosystems; Dharmacon Inc. For MPZL1 knockdown, the shRNA sequences were: $M P Z L 1$-shRNA-1, 5'-TGACATCAC AGATATAGGT-3'; MPZL1-shRNA-2, 5'-TCAAGTGGCATA GCCAATG-3'; and shRNA-negative control (NC), 5'-ACC TCCACCCTCACTCTGCCAT-3'. For MPZL1 overexpression, the coding sequence of $M P Z L 1$ was inserted into the lentiviral vector pLenti 7.3.

For $S r c$ knockdown, $S r c$-small interfering RNA (siRNA) was used at a final concentration of $25 \mathrm{nM}$ and was transfected into cells (60\% confluent) using Lipofectamine ${ }^{\circledR}$ RNAiMAX reagent (Invitrogen; Thermo Fisher Scientific, Inc.) for $72 \mathrm{~h}$ at $37^{\circ} \mathrm{C}$, according to the manufacturer's protocol. For Src knockdown, the siRNA sequences (Shanghai GenePharma Co., Ltd.) were: $S r c$-siRNA, 5'-CAGGCUGAGGAGUGGUAUUTT-3'; and siRNA-NC, 5'-TTCTCCGAACGTGTCACGT-3'.
Western blotting. Cells were lysed in RIPA buffer [Tris (pH 7.4), $50 \mathrm{mM}$; NaCl, $150 \mathrm{mM}$; $1 \% \mathrm{NP}-40 ; 0.1 \%$ SDS; EDTA, $2 \mu \mathrm{M}$ ] containing proteinase inhibitors (Roche Molecular Diagnostics) and phosphatase inhibitors (Roche Molecular Diagnostics), and the protein concentration was determined using the bicinchoninic acid assay. The cell lysates $(20 \mu \mathrm{g}$ total protein) were subjected to 8-10\% SDS-PAGE and immunoblotting. Subsequently, proteins were transferred to nitrocellulose membranes, which were blocked for $1 \mathrm{~h}$ with $5 \%$ nonfat milk at room temperature, and were then incubated with primary antibodies $(1: 1,000)$ at $4^{\circ} \mathrm{C}$ overnight. The membranes were then incubated for $1 \mathrm{~h}$ with horseradish peroxidase-linked anti-rabbit IgG antibody (1:1,000; cat. no. 7074; Cell Signaling Technology, Inc) at room temperature. Blots were visualized with ECL western blotting reagents (Thermo Fisher Scientific, Inc.) using ChemiDoc XRS+ (Bio-Rad Laboratories, Inc.). Semi-quantification was conducted using ImageLab 2.0 software Bio-Rad Laboratories, Inc.). Antibodies against the following proteins were used: p130 (cat. no. 13846), phosphorylated (p)-p130 (cat. no. 4015), cortactin (cat. no. 3502), p-cortactin (cat. no. 4569), Src (cat. no. 2108), p-Src (cat. no. 12432), MPZL1 (cat. no. 9893) and GAPDH (cat. no. 5174) (all Cell Signaling Technology, Inc). Notably, since MPZL1 has three alternatively spliced isoforms, at least three bands can be seen in MPZL1 blots, and all bands were measured when semi-quantifying the blots.

Cell proliferation assay. Cell proliferation was assessed using the Cell Counting Kit-8 (CCK-8; Dojindo Molecular Technologies, Inc.). Cells (5,000 cells/well) were seeded in triplicate in 96-well plates. After 24, 48, 72, 96 or $120 \mathrm{~h}$ at $37^{\circ} \mathrm{C}$, CCK-8 reagent ( $1 / 20$, volume/volume) was added to the cells for $2 \mathrm{~h}$ at $37^{\circ} \mathrm{C}$. The absorbance of each well was measured at $450 \mathrm{~nm}$, according to the manufacturer's protocol.

Cell migration and invasion assays. Transwell chambers with $8 \mu \mathrm{m}$ pore membranes (Corning, Inc.) were placed in 24-well culture plates and were incubated with serum-free RPMI- 1640 medium at $37^{\circ} \mathrm{C}$ for $1 \mathrm{~h}$. A $200 \mu \mathrm{l}$ suspension of $0.5-1 \times 10^{5}$ cells was seeded into the upper compartment of the Transwell chambers in FBS-free medium, whereas 600-800 $\mu \mathrm{l}$ RPMI-1640 medium supplemented with 10\% FBS was added into the bottom wells; cells were incubated at $37^{\circ} \mathrm{C}$ for $12-24 \mathrm{~h}$. The migrating cells that were attached to the lower membranes of the Transwell chambers were stained with crystal violet $(0.1 \%)$ at room temperature for $2 \mathrm{~h}$ and images were captured at x200 magnification under a light microscope. Images of three random fields from three replicate wells were obtained, and the migrated cells were counted. The cell invasion assay was the same as the migration assay; however, the chambers were coated with Matrigel.

Colony formation assay. Cells were seeded in triplicate in 6-well plates. After 12 days, the cells were washed with PBS, fixed with methanol for $1 \mathrm{~h}$ and stained with $0.1 \%$ crystal violet for $1 \mathrm{~h}$ at room temperature. Subsequently, images of the colonies were captured and counted.

Immunohistochemistry. Ovarian cancer specimens were collected from the 78 patients during surgery at Tongren 
Table I. Association between clinicopathological characteristics and MPZL-1 expression.

\begin{tabular}{|c|c|c|c|c|c|c|}
\hline \multirow[b]{3}{*}{ Variable } & \multirow[b]{3}{*}{ Cases (n) } & \multicolumn{4}{|c|}{ MPZL-1 expression score } & \multirow[b]{3}{*}{ P-value } \\
\hline & & \multicolumn{2}{|c|}{ Low level } & \multicolumn{2}{|c|}{ High level } & \\
\hline & & - & + & ++ & +++ & \\
\hline \multicolumn{7}{|c|}{ Age (years) } \\
\hline$<55$ & 28 & 1 & 8 & 9 & 10 & \\
\hline$\geq 55$ & 18 & 0 & 4 & 4 & 10 & 0.69 \\
\hline \multicolumn{7}{|c|}{ Clinical stage } \\
\hline I-II & 9 & 1 & 5 & 2 & 1 & \\
\hline III & 25 & 0 & 5 & 8 & 12 & $0.01^{\mathrm{a}}$ \\
\hline IV & 12 & 0 & 2 & 3 & 7 & $0.02^{\mathrm{b}}$ \\
\hline \multicolumn{7}{|c|}{ CA125 (U/ml) } \\
\hline$\leq 200$ & 33 & 1 & 12 & 10 & 10 & \\
\hline$>200$ & 13 & 0 & 0 & 3 & 10 & 0.01 \\
\hline
\end{tabular}

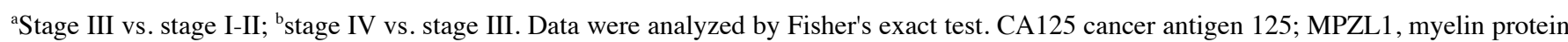
zero like 1.

Hospital, Shanghai Jiao Tong University. The tissue specimens were fixed in $4 \%$ paraformaldehyde (Beijing Solarbio Science \& Technology Co., Ltd.) for $24 \mathrm{~h}$ at room temperature and embedded in paraffin, before being cut into $5 \mu \mathrm{m}$ sections. The tissue sections were deparaffinized, treated with $3 \% \mathrm{H}_{2} \mathrm{O}_{2}$ for $10 \mathrm{~min}$ at room temperature, autoclaved in $10 \mathrm{mM}$ citric sodium ( $\mathrm{pH}$ 6.0) for 30 min to unmask antigens and rinsed in PBS. Subsequently, sections were incubated with primary antibodies against MPZL1 (1:200; cat. no. GTX46451; GeneTex, Inc.) at $4^{\circ} \mathrm{C}$ overnight, followed by incubation with a biotinylated secondary antibody (1:1,000; cat. no. ab6844; Abcam) for $1 \mathrm{~h}$ at room temperature. Signal amplification and detection was performed using the DAB system (Dako) according to the manufacturer's instructions.

Immunohistochemistry scoring. All cases were analyzed independently with the help of two expert pathologists and were assigned a score according to the following criteria: Strongly positive, $3+$; positive, $2+$; weakly positive, $1+$; and negative, 0 .

Statistical analysis. Statistical analysis was performed using GraphPad Prism 6 software (GraphPad Software, Inc.). Genomic analysis of MPZL1 in ovarian cancer (489 high-grade serous ovarian cancer specimens were surgically resected prior to systemic treatment; all patients received a platinum agent and $94 \%$ received a taxane) was performed with The Cancer Genome Atlas (TCGA) copy number portal (www.broadinstitute.org/tcga). In all experiments, comparisons between two groups were conducted using two-sided Student's t-test, and one-way analysis of variance followed by Tukey's multiple comparisons test was used to test for differences among more groups. Fisher's exact test was used to determine differences between stages, as presented in Table I. $\mathrm{P}<0.05$ was considered to indicate a statistically significant difference.

\section{Results}

Overexpression of MPZL1 is associated with malignant features of ovarian cancer. Copy number analysis of TCGA ovarian serous adenocarcinoma samples in the cBioportal database revealed that MPZL1, located at chromosome 1q24 (magnified section in Fig. 1A), was genomically amplified in a considerable proportion of cases (Fig. 1A). Notably, the expression levels of MPZL1 were associated with its amplification status (Fig. 1B), thus indicating that the high expression of MPZL1 in tumor tissues may be regulated by copy number amplification of the MPZL1 gene. To further determine the association of MPZL1 expression with the malignant features of ovarian cancer, MPZL1 protein expression was assessed by immunohistochemistry among benign, borderline and malignant epithelial ovarian cancer patient tissues (Fig. 1C; Table I). The results revealed that MPZL1 expression was almost undetectable in patients with benign ovarian cancer, whereas it was expressed at significantly higher levels in malignant cancer patient tissues compared with in benign/borderline cancer patient tissues (Fig. 1D). In addition, the expression profile of the MPZL1 protein was determined using The Human Protein Atlas (18). MPZL1 was ubiquitously expressed in different human tissues as well as various cancer types. In normal ovarian tissues, MPZL1 was lowly expressed, whereas in some ovarian cancer tissues, MPZL1 was overexpressed (data not shown). Taken together, these data indicated that MPZL1 was overexpressed in a subset of patients with ovarian cancer, suggesting that MPZL1 may serve a pivotal role in ovarian cancer.

Overexpression of MPZL1 promotes ovarian cancer cell proliferation and migration. To study the biological function of MPZL1, the protein expression levels of MPZL1 
A

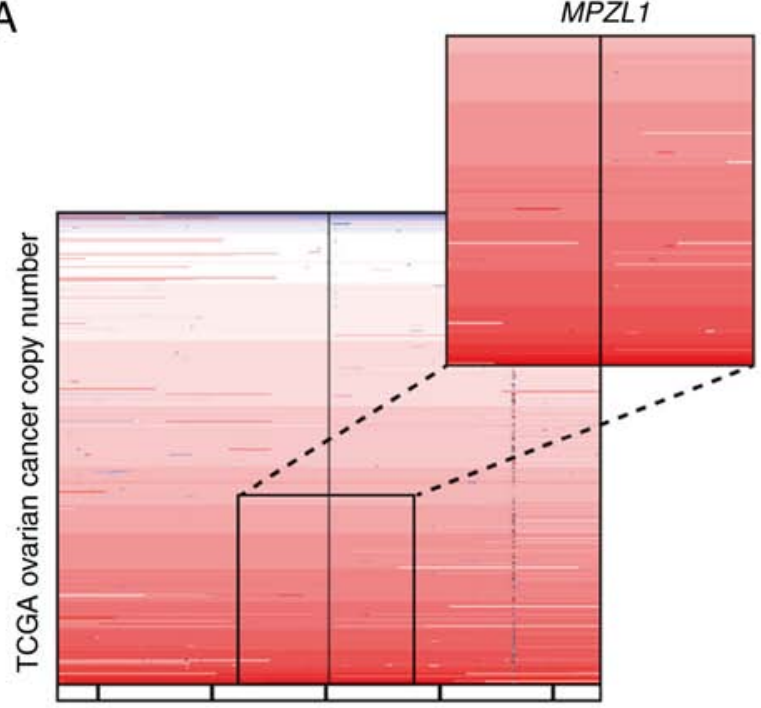

Chr1 $164 \mathrm{Mb} 165 \mathrm{Mb} 166 \mathrm{Mb} 167 \mathrm{Mb} 168 \mathrm{Mb}$

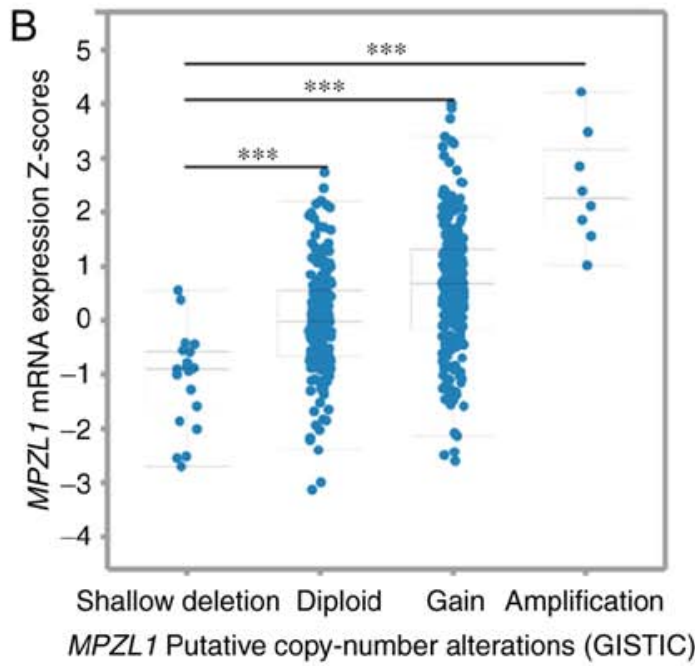

C

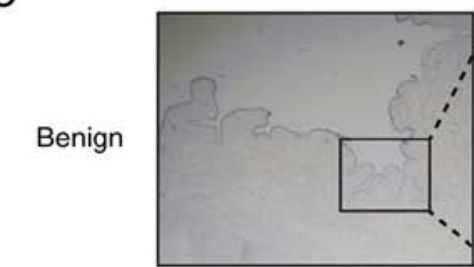

Patient 2

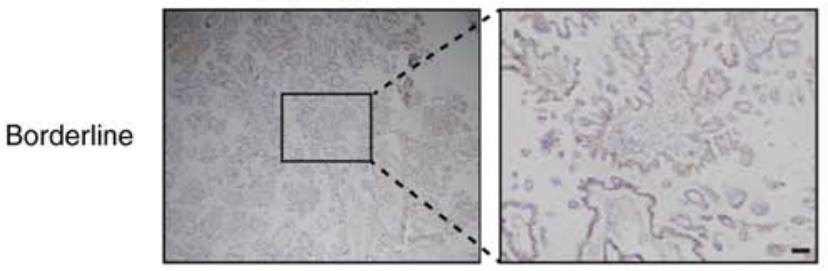

Patient 3

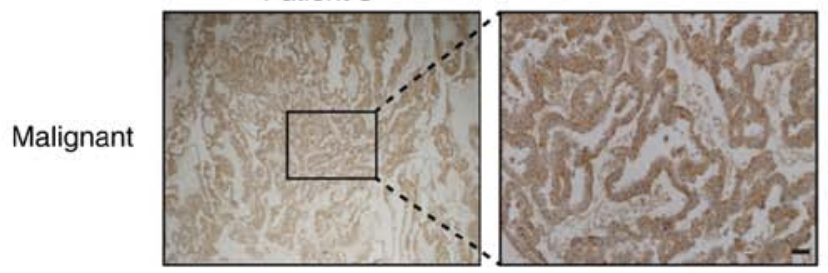

D

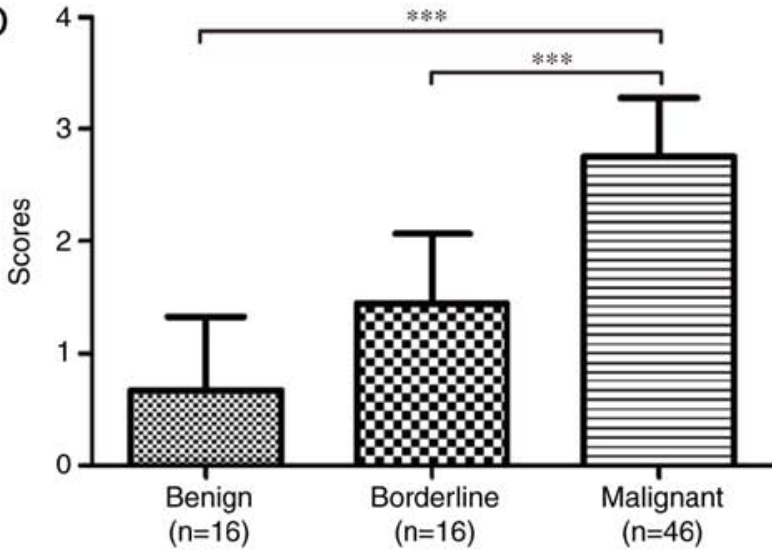

Figure 1. Overexpression of MPZL1 is associated with ovarian cancer. (A) Copy number analysis of $M P Z L 1$ in TCGA ovarian cancer samples. Color scale: Red, amplification; blue, deletion. (B) $M P Z L 1$ gene expression in ovarian cancer with different $M P Z L 1$ copy number alterations. (C) Representative images of immunohistochemical staining of the MPZL1 protein in human ovarian cancer samples (scale bar, $50 \mu \mathrm{m}$ ). (D) Histogram of the relative immunohistochemistry scores of MPZL1 in the three ovarian cancer groups (benign, $n=16$; borderline, $n=16$; malignant, $n=46$ ). Data are presented as the mean \pm SEM.

${ }^{* * *} \mathrm{P}<0.0001$. GISTIC, Genomic Identification of Significant Targets in Cancer; MPZL1, myelin protein zero like 1; TCGA, The Cancer Genome Atlas.

were detected in four ovarian cancer cell lines (Fig. 2A). Subsequently, the MPZL1 gene was overexpressed via lentiviral infection in SKOV3 and TOV-21G cells (Fig. 2B); these two cell lines were selected as they exhibited relatively lower endogenous MPZL1 expression. Subsequently, the effects of MPZL1 overexpression on the proliferation of these ovarian cancer cells were determined using the CCK- 8 assay. Notably, the results demonstrated that exogenous overexpression of the MPZL1 gene promoted ovarian cancer cell proliferation (Fig. 2C). Similarly, the results of the colony formation assay demonstrated that the number of colonies was significantly increased in MPZL1-overexpressed SKOV3 and TOV-21G cells (Fig. 2D). Furthermore, the effects of MPZL1 overexpression were examined on the migratory and invasive abilities of ovarian cancer cells by Transwell assays; ectopic expression of MPZL1 significantly enhanced the in vitro migration and invasion of ovarian cancer cells (Fig. 2E). These results indicated that MPZL1 may serve an important role in promoting cell migration and metastasis of ovarian cancer.

Targeted downregulation of MPZL1 attenuates ovarian cancer cell proliferation and migration. To further verify the role of MPZL1 in ovarian cancer cell migration, HO8910 and HEY cells were selected as cellular models for loss-of-function studies. Firstly, the MPZL1 gene was stably knocked down in HO8910 and HEY cells using a lentiviral shRNA specifically targeting MPZL1 (Fig. 3A). Consequently, cell proliferation was significantly inhibited under normal growth conditions (Fig. 3B). Consistently, the colony formation assay revealed that the number of colonies was evidently decreased following knockdown of MPZL1 in HO8910 and HEY cells (Fig. 3C). Furthermore, Transwell assays demonstrated that the in vitro migration and invasion of ovarian cancer cells 

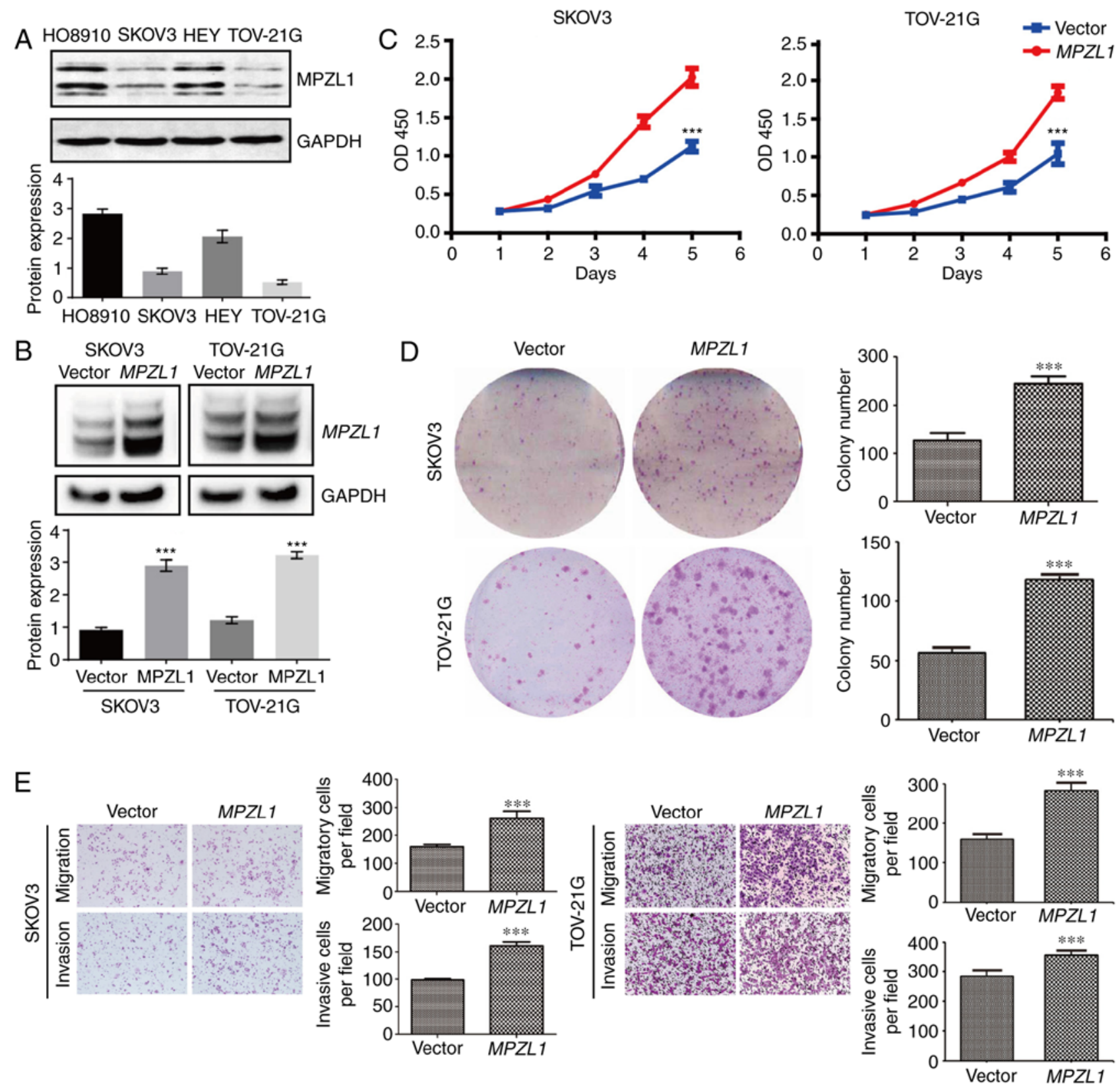

Figure 2. Overexpression of $M P Z L 1$ promotes ovarian cancer cell proliferation and migration. (A) Detection of $M P Z L 1$ protein expression in four ovarian cancer cell lines by western blotting. (B) Detection of lentivirus-mediated overexpression of MPZL1 in SKOV3 and TOV-21G cells by western blotting. (C) MPZL1 was overexpressed in SKOV3 and TOV-21G cells. Cell proliferation was analyzed by Cell Counting Kit-8. Error bars indicate standard deviation. (D) Colony formation assays in SKOV3 and TOV-21G cells (magnification, x200). (E) Representative results of the Transwell assays to determine the effects of MPZL1 overexpression on the in vitro migratory and invasive abilities of SKOV3 and TOV-21G cells (magnification, $\mathrm{x} 200$ ). Each group consisted of three biological replicates, and data are presented as the mean $\pm \mathrm{SEM} .{ }^{* * *} \mathrm{P}<0.001$ vs. vector. MPZL1, myelin protein zero like 1 ; OD, optical density.

were inhibited by MPZL1 depletion (Fig. 3D). These findings indicated that MPZL1 knockdown attenuated proliferation, migration and invasion of ovarian cancer cells.

MPZL1 regulates phosphorylation levels of numerous pro-metastatic proteins. Recently, many studies have reported that pro-metastatic proteins, including p130, Src and cortactin, are important for cell migration and tumor metastasis (19-21). Furthermore, p130 and cortactin were originally identified as substrate proteins of the Src family kinases, and a previous report demonstrated that the MPZL1/Src/cortactin signaling cascade functions in the process of hepatocellular carcinoma cell migration (22).
However, to the best of our knowledge, the potential role of MPZL1 in the phosphorylation of pro-metastatic proteins has not been determined in ovarian cancer. To investigate whether the MPZL1/Src/cortactin signaling cascade exists in ovarian cancer, the expression levels of pro-metastatic proteins were detected by western blotting. The results indicated that stable overexpression of MPZL1 led to increased phosphorylation of p130, Src and cortactin in SKOV3 cells (Fig. 4A). Furthermore, targeted knockdown of the MPZL1 gene by shRNA reduced phosphorylation of these three proteins in HO8910 cells (Fig. 4B). These findings indicated that MPZL1 overexpression reprogrammed the pro-metastatic signaling network in ovarian cancer. 

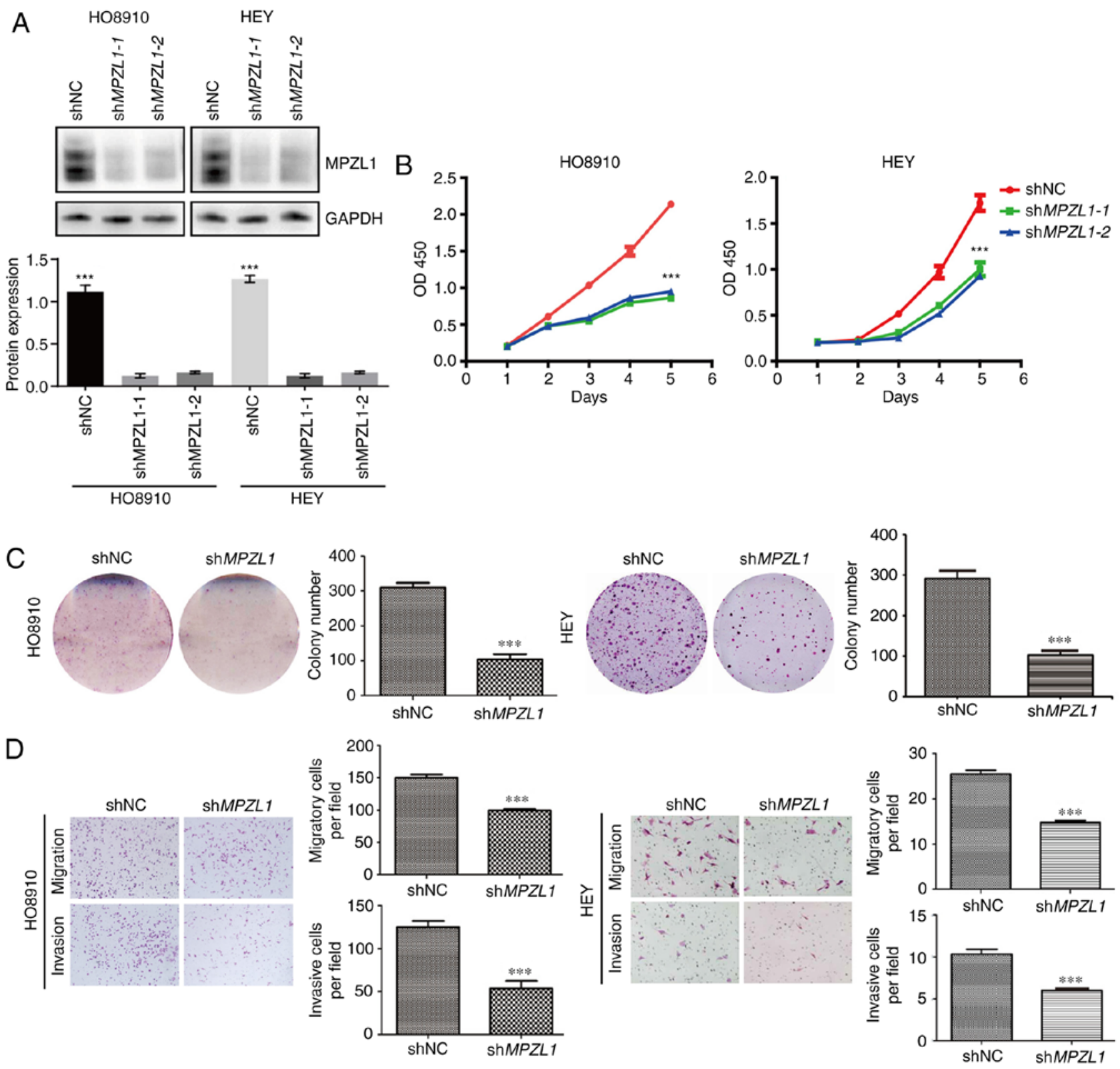

Figure 3. Targeted downregulation of MPZL1 attenuates ovarian cancer cell proliferation and migration. (A) Detection of knockdown of MPZL1 by shRNA in HO8910 and HEY cells by western blotting. (B) MPZL1 was knocked down by shRNA in HO8910 and HEY cells, and cell proliferation was analyzed by Cell Counting Kit-8. Error bars indicate standard deviation. Data are presented as the mean \pm SEM. ${ }^{* * *} \mathrm{P}<0.001$ vs. shNC. (C) Colony formation assays in HO8910 and HEY cells (magnification, x200). (D) Representative results of the Transwell assays to determine the effects of MPZL1 knockdown on the in vitro migratory and invasive abilities of HO8910 and HEY cells (magnification, $\mathrm{x} 200$ ). Each group consisted of three biological replicates. Data are presented as the mean \pm SEM. ${ }^{* * *} \mathrm{P}<0.001$ vs. shNC. MPZL1, myelin protein zero like 1; NC, negative control; OD, optical density; sh/shRNA, short hairpin RNA.

Transient transfection of Src siRNA suppresses migration and invasion in SKOV3-MPZL1 cells. According to previous studies, Src tyrosine kinases serve critical roles in several cellular signal transduction pathways that regulate cell proliferation, adhesion, migration and invasion (22-25). Additionally, as substrate proteins of Src kinase, p-p130 and p-cortactin participate in cell migration (26). Furthermore, amplification of MPZL1 promotes tumor cell migration via Src-mediated cortactin phosphorylation in hepatocellular carcinoma cells (27). To ascertain whether Src kinase activity is essential for MPZL1-induced phosphorylation of p130 and cortactin in ovarian cancer cells, MPZL1 was overexpressed in SKOV3 cells, and the $S r c$ gene was then knocked down by siRNA. Notably, stable overexpression of MPZL1 significantly increased phosphorylation of p130, cortactin and Src in SKOV3-MPZL1 cells, and enhanced the migratory and invasive abilities of SKOV3-MPZL1 cells (Fig. 5A-C). Conversely, targeted knockdown of $\operatorname{Src}$ via siRNA resulted in reduced phosphorylation of p130 and cortactin, and suppressed the migration and invasion of SKOV3-MPZL1 cells (Fig. 5A-C). Taken together, these findings indicated that Src kinase activity may be essential for MPZL1-mediated migration and invasion of ovarian cancer cells.

\section{Discussion}

In the present study, amplified MPZL1 was associated with the malignant features of ovarian cancer. Subsequently, 
A
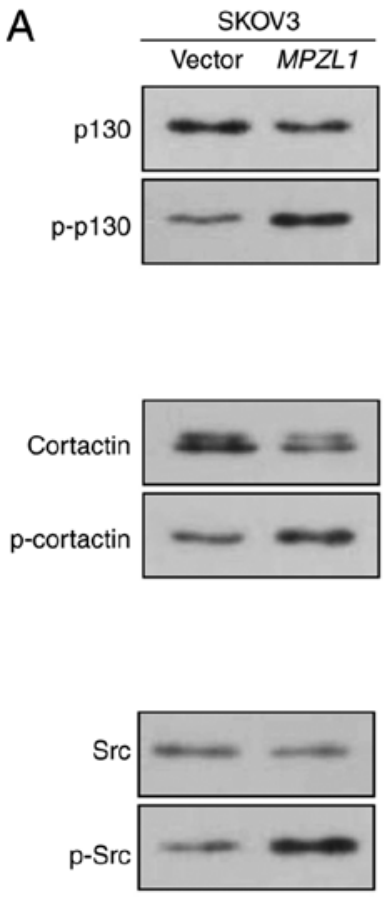

p-Src
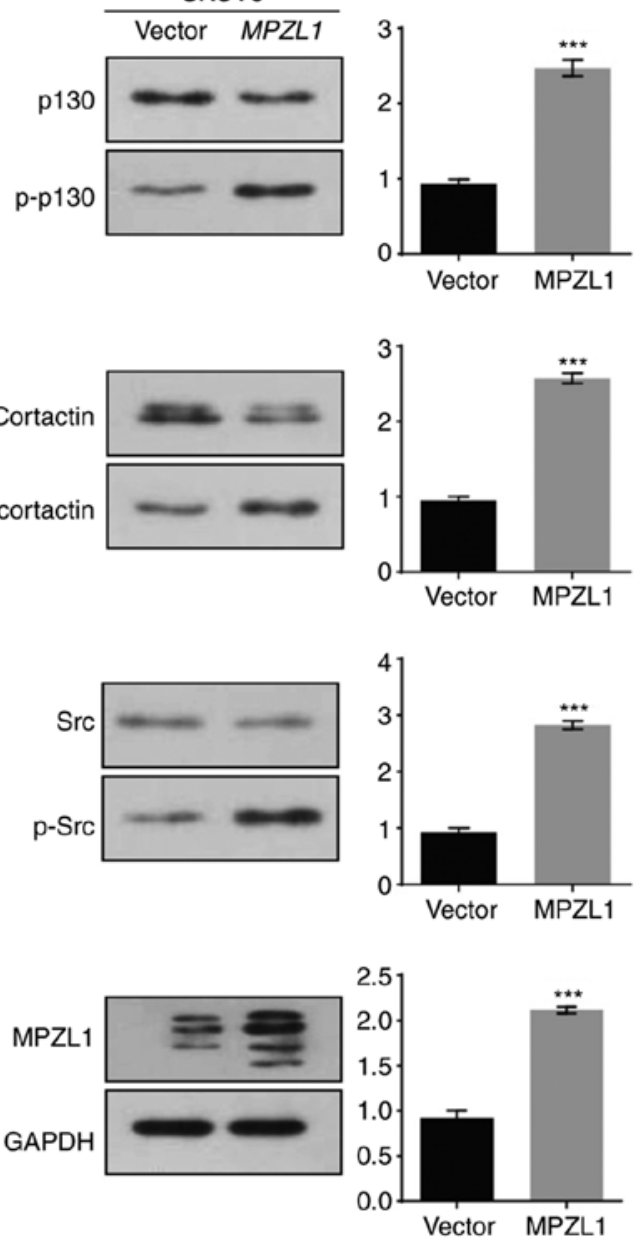

B
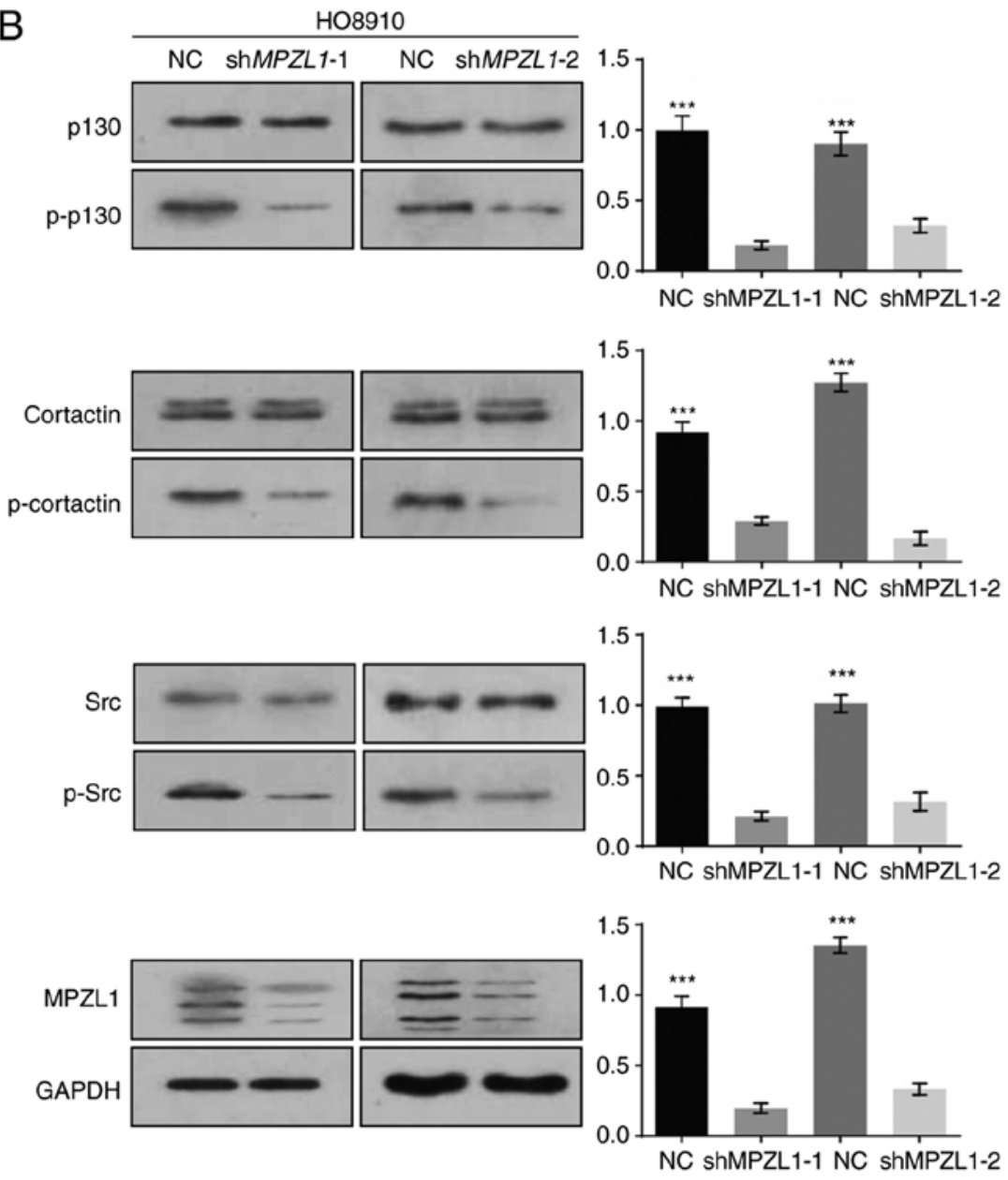

Figure 4. MPZL1 regulates the phosphorylation of numerous pro-metastatic proteins. (A) Overexpression of MPZL1 in SKOV3 cells increased the phosphorylation of p130, cortactin and Src. ${ }^{* * *} \mathrm{P}<0.001$ vs. vector. (B) Knockdown of MPZL1 by shRNA in HO8910 cells decreased the phosphorylation of p130, cortactin and Src. Phosphorylated proteins were normalized to both total proteins and GAPDH. Each group consisted of three biological replicates and representative images are shown. Data are presented as the mean \pm SEM. ${ }^{* * *} \mathrm{P}<0.001$ vs. NC. MPZL1, myelin protein zero like 1; NC, negative control; $p-$, phosphorylated; sh/shRNA, short hairpin RNA.

overexpression and knockdown of MPZL1 indicated that MPZL1 served a prominent role in the promotion of ovarian cancer cell proliferation, migration and invasion. In addition, amplification of $M P Z L 1$ promoted tumor cell migration through Src-mediated phosphorylation of p130 and cortactin. Therefore, these data suggested that MPZL1 may operate as a novel oncoprotein in ovarian cancer.

Previous studies have reported that the MPZL1 protein is involved in cell signaling, proliferation, differentiation and transformation $(15,28)$. Furthermore, amplification of MPZL1 promotes tumor cell migration through Src-mediated phosphorylation of cortactin in hepatocellular carcinoma $(27,29)$. However, the biological functions and clinical implications of MPZL1 in other types of human cancer remain unclear. This study identified a positive association between the protein expression levels of MPZL1 and cell proliferation, migration and invasion of ovarian cancer cells.

Src family protein tyrosine kinases participate in numerous signaling pathways that control cellular responses, including proliferation, survival, adhesion and migration in normal and cancer cells (30-33). As substrate proteins of Src kinase, p130 and cortactin become tyrosine-phosphorylated during integrin-mediated cell adhesion to the extracellular matrix, cell attachment and cell invasion (24,34-36). Furthermore, it has been demonstrated that tyrosine-phosphorylated cortactin via Src kinase, which is activated upon MPZL1 overexpression, increases the migratory potential of hepatocellular carcinoma cells $(14,27)$. In this study, it was demonstrated that overexpression of MPZL1 resulted in increased phosphorylation of Src, p130 and cortactin. Additionally, the migration and proliferation of ovarian cancer cells were promoted. These findings indicated that MPZL1 may serve an important role in ovarian cancer cell metastasis. However, the physiological relevance of these data requires further mechanistic investigations and in vivo studies.

In conclusion, the present results suggested that amplification of MPZL1 in ovarian cancer may be associated with the malignant features of ovarian cancer. MPZL1 was involved in Src-mediated phosphorylation of p130 and cortactin, thereby promoting ovarian cancer cell proliferation, migration and invasion. Furthermore, these data identifies MPZL1 as a novel pro-metastatic gene in ovarian cancer. Together with a report implicating MPZL1 in other cancer types (27), the present study expands our understanding of human ovarian cancer metastasis and indicates potential therapeutic avenues for the treatment of ovarian cancer. 
A
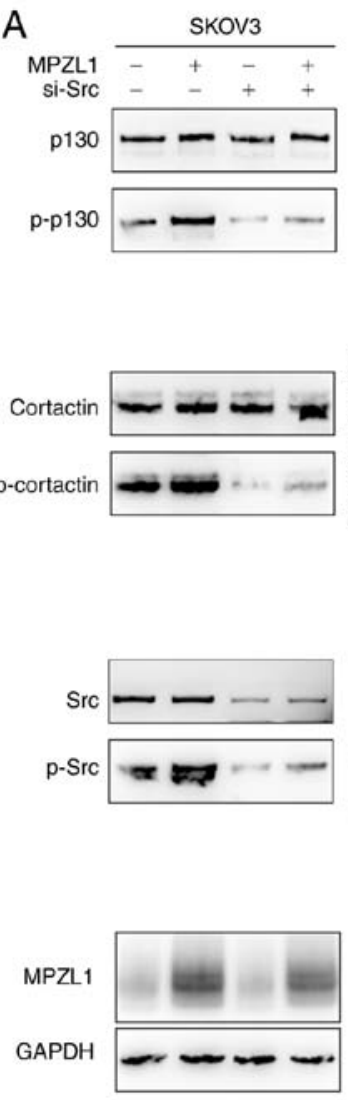
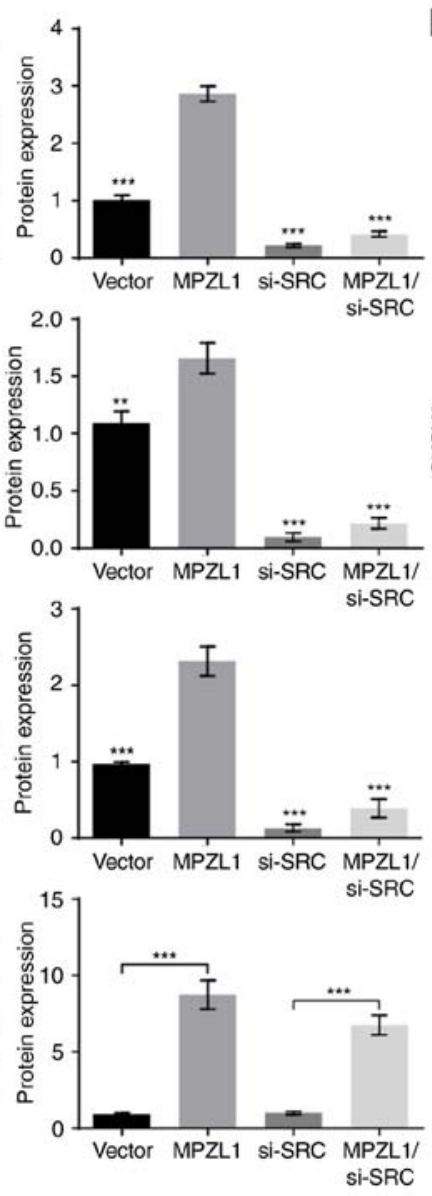

B

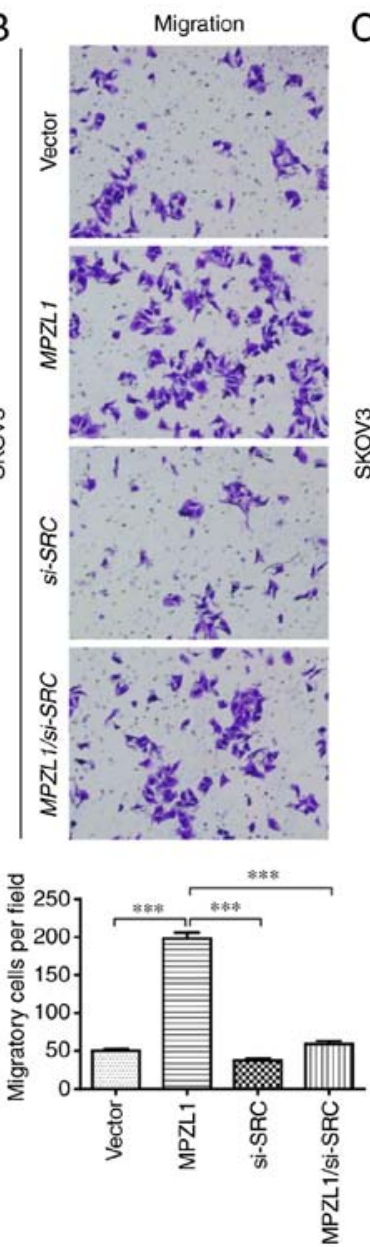

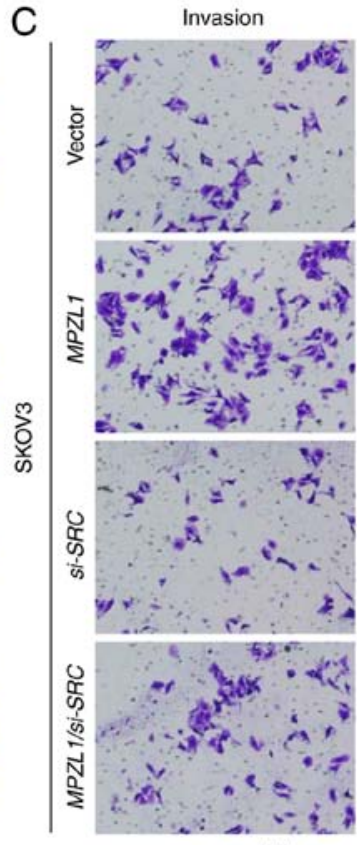

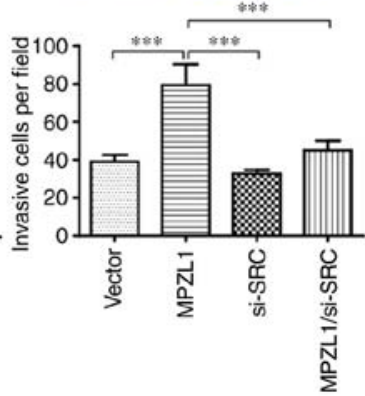

Figure 5. Transient transfection of Src siRNA decreases cell migration and invasion in SKOV3-MPZL1 cells. (A) Western blot analysis following transient transfection of Src siRNA in SKOV3-MPZL1 cells; control cells were transfected with NC siRNA. Phosphorylated proteins were normalized to both total proteins and GAPDH. (B) Transwell migration assays of SKOV3 cells (magnification, x200). (C) Transwell invasion assays of SKOV3 cells (magnification, $\mathrm{x} 200)$. Each group consisted of three biological replicates. Data are presented as the mean $\pm \mathrm{SEM} .{ }^{* *} \mathrm{P}<0.01,{ }^{* * * *} \mathrm{P}<0.001$ vs. MPZL1 or as indicated. MPZL1, myelin protein zero like 1; p-, phosphorylated; siRNA, small interfering RNA.

\section{Acknowledgements}

The authors would like to thank Dr Shengzhe Zhang and Ms. Zhenfeng Zhang (Shanghai Jiao Tong University School of Medicine) for their technical assistance.

\section{Funding}

This work was supported by a grant from the Science and Technology Commission of Changning District, Shanghai (grant no. CNKW2017Y08 to X.W.).

\section{Availability of data and materials}

The datasets used and/or analyzed during the current study are available from the corresponding author on reasonable request.

\section{Authors' contributions}

XW and DC designed and conceived the experiments. LC analyzed the data. XW, DC and LC drafted the manuscript and wrote the manuscript. XW supervised the study. All authors read and approved the final manuscript.

\section{Ethics approval and consent to participate}

All experimental protocols were approved by the Ethics Committee of the Tongren Hospital, Shanghai Jiao Tong University School of Medicine. All patients provided written informed consent.

\section{Patient consent for publication}

Not applicable.

\section{Competing interests}

The authors declare that they have no competing interests.

\section{References}

1. Marcus CS, Maxwell GL, Darcy KM, Hamilton CA and McGuire WP: Current approaches and challenges in managing and monitoring treatment response in ovarian cancer. J Cancer 5: 25-30, 2014.

2. Oronsky B, Ray CM, Spira AI, Trepel JB, Carter CA and Cottrill HM: A brief review of the management of platinumresistant-platinum-refractory ovarian cancer. Med Oncol 34: 103, 2017. 
3. Walker JL, Powell CB, Chen Lm, Carter J, Bae Jump VL, Parker LP, Borowsky ME and Gibb RK: Society of gynecologic oncology recommendations for the prevention of ovarian cancer. Cancer 121: 2108-2120, 2015

4. Matz M, Coleman MP, Carreira H, Salmerón D, Chirlaque MD and Allemani C; CONCORD Working Group: Worldwide comparison of ovarian cancer survival: Histological group and stage at diagnosis (CONCORD-2). Gynecol Oncol 144: 396-404, 2017.

5. Jiménez-Sánchez A, Memon D, Pourpe $\mathrm{S}$, Veeraraghavan $\mathrm{H}$, Li Y, Vargas HA, Gill MB, Park KJ, Zivanovic O, Konner J, et al: Heterogeneous tumor-immune microenvironments among differentially growing metastases in an ovarian cancer patient. Cell 170: 927-938, 2017

6. Hillman RT, Lu KH and Futreal PA: A novel genomic rearrangement signature to predict poor survival among women with high grade serous ovarian cancer. J Clin Oncol 35 (Suppl 15): S5509, 2017.

7. Lengyel E: Ovarian cancer development and metastasis. Am J Pathol 177: 1053-1064, 2010.

8. Bast RC, Hennessy B and Mills GB: The biology of ovarian cancer: New opportunities for translation. Nat Rev Cancer 9: 415-428, 2009.

9. Naora H and Montell DJ: Ovarian cancer metastasis: Integrating insights from disparate model organisms. Nat Rev Cancer 5: 355-366, 2005.

10. Luo Z, Wang Q, Lau WB, Lau B, Xu L, Zhao L, Yang H, Feng M, Xuan Y, Yang Y, et al: Tumor microenvironment: The culprit for ovarian cancer metastasis? Cancer Lett 377: 174-182, 2016.

11. He G, Liu X, Qin W, Chen Q, Wang X, Yang Y, Zhou J, Xu Y, Gu N, Feng G, et al: MPZL1/PZR, a novel candidate predisposing schizophrenia in Han Chinese. Mol Psychiatry 11: 748-751, 2006.

12. Beigbeder A, Chartier FJ and Bisson N: MPZL1 forms a signalling complex with GRB2 adaptor and PTPN11 phosphatase in HER2-positive breast cancer cells. Sci Rep 7: 11514, 2017.

13. Zhao R, Guerrah A, Tang $\mathrm{H}$ and Zhao ZJ: Cell surface glycoprotein PZR is a major mediator of concanavalin A-induced cell signaling. J Biol Chem 277: 7882-7888, 2002.

14. Fonseca NA, He Y, Greger L, Brazma A and Zhang Z: Comprehensive genome and transcriptome analysis reveals genetic basis for gene fusions in cancer. bioRxiv: 148684, 2017.

15. Zhao ZJ and Zhao R: Purification and cloning of PZR, a binding protein and putative physiological substrate of tyrosine phosphatase SHP-2. J Biol Chem 273: 29367-29372, 1998.

16. Playford MP and Schaller MD: The interplay between Src and integrins in normal and tumor biology. Oncogene 23: 7928-7946, 2004

17. Wang L, Dong P, Zhang Y, Yang M, Chen Y and Tian BL: Prognostic validation of the updated 8th edition Tumor-NodeMetastasis classification by the Union for international cancer control: Survival analyses of 307 patients with surgically treated gallbladder carcinoma. Oncol Lett 16: 4427-4433, 2018.

18. Uhlen M, Oksvold P, Fagerberg L, Lundberg E, Jonasson K, Forsberg M, Zwahlen M, Kampf C, Wester K, Hober S, et al: Towards a knowledge-based human protein atlas. Nature Biotechnol 28: 1248-1250, 2010.

19. Dai Y, Qi L,Zhang X, Li Y, Chen M and Zu X: CrkI and p130(Cas) complex regulates the migration and invasion of prostate cancer cells. Cell Biochem Funct 29: 625-629, 2011.

20. Padhye A, Ungewiss C, Fradette JJ, Rodriguez BL, Albritton JL, Miller JS and Gibbons DL: A novel ex vivo tumor system identifies Src-mediated invasion and metastasis in mesenchymal tumor cells in non-small cell lung cancer. Sci Rep 9: 4819, 2019.
21. Wu H, Jing X, Cheng X, He Y, Hu L, Wu H, Ye F and Zhao R: Asporin enhances colorectal cancer metastasis through activating the EGFR/src/cortactin signaling pathway. Oncotarget 7: 73402-73413, 2016.

22. Zhang XH, Wang Q, Gerald W, Hudis CA, Norton L, Smid M, Foekens JA and Massagué J: Latent bone metastasis in breast cancer tied to Src-dependent survival signals. Cancer cell 16: 67-78, 2009.

23. Summy JM and Gallick GE: Src family kinases in tumor progression and metastasis. Cancer Metastasis Rev 22: 337-358, 2003.

24. Huang J, Asawa T, Takato T and Sakai R: Cooperative roles of fyn and cortactin in cell migration of metastatic murine melanoma. J Biol Chem 278: 48367-48376, 2003.

25. Brábek J, Constancio SS, Siesser PF, Shin NY, Pozzi A and Hanks SK: Crk-associated substrate tyrosine phosphorylation sites are critical for invasion and metastasis of SRC-transformed cells. Mol Cancer Res 3: 307-315, 2005.

26. Parsons SJ and Parsons JT: Src family kinases, key regulators of signal transduction. Oncogene 23: 7906-7909, 2004

27. Jia D, Jing Y, Zhang Z, Liu L, Ding J, Zhao F, Ge C, Wang Q, Chen T, Yao M, et al: Amplification of MPZL1/PZR promotes tumor cell migration through src-mediated phosphorylation of cortactin in hepatocellular carcinoma. Cell Res 24: 204-217, 2014.

28. Zhao R and Zhao ZJ: Dissecting the interaction of SHP-2 with PZR, an immunoglobulin family protein containing immunoreceptor tyrosine-based inhibitory motifs. J Biol Chem 275: $5453-5459,2000$

29. Yeh YT, Dai HY and Chien CY: Amplification of MPZL1/PZR gene in hepatocellular carcinoma. Hepatobiliary surg Nutr 3: 87-90, 2014

30. Giannoni E, Buricchi F, Raugei G, Ramponi G and Chiarugi P: Intracellular reactive oxygen species activate Src tyrosine kinase during cell adhesion and anchorage-dependent cell growth. Mol Cell Biol 25: 6391-6403, 2005.

31. Shor AC, Keschman EA, Lee FY, Muro-Cacho C, Letson GD, Trent JC, Pledger WJ and Jove R: Dasatinib inhibits migration and invasion in diverse human sarcoma cell lines and induces apoptosis in bone sarcoma cells dependent on SRC kinase for survival. Cancer Res 67: 2800-2808, 2007.

32. Yu H and Jove R: The STATs of cancer-new molecular targets come of age. Nat Rev Cancer 4: 97-105, 2004.

33. Mitra SK and Schlaepfer DD: Integrin-regulated FAK-src signaling in normal and cancer cells. Curr Opin Cell Biol 18: 516-523, 2006.

34. Vuori K and Ruoslahti E: Tyrosine phosphorylation of p130Cas and cortactin accompanies integrin-mediated cell adhesion to extracellular matrix. J Biol Chem 270: 22259-22262, 1995.

35. Ammer AG, Kelley LC, Hayes KE, Evans JV, Lopez-Skinner LA, Martin KH, Frederick B, Rothschild BL, Raben D, Elvin P, et al: Saracatinib impairs head and neck squamous cell carcinoma invasion by disrupting invadopodia function. J Cancer Sci Ther 1: 52-61, 2009.

36. Agerer F, Lux S, Michel A, Rohde M, Ohlsen K and Hauck CR: Cellular invasion by Staphylococcus aureus reveals a functional link between focal adhesion kinase and cortactin in integrin-mediated internalisation. J Cell Sci 118: 2189-2200, 2005.

This work is licensed under a Creative Commons Attribution-NonCommercial-NoDerivatives 4.0 International (CC BY-NC-ND 4.0) License. 